Talal Hilal

MBBS

University of Kentucky, Lexington, Ky, USA

talal.hilal@uky.edu

doi: 10.5694/mjal5.00372

\title{
Snapshot
}

\section{Bilateral upper lobe opacification}

A

29-year-old Hispanic man presented with a 2-month history of severe progressive dyspnoea, productive cough and $6.8 \mathrm{~kg}$ weight loss. His social history was significant: from 1995 to 2005 he had worked in a factory sand-blasting denim while wearing ill-fitting, reportedly ineffective protective clothing. His symptoms had slowly progressed since they commenced in 2008.

Chest auscultation identified bronchial breath sounds with crackles in the upper zones. Chest x-ray showed bilateral upper lung opacification representing extensive pulmonary consolidation and fibrosis (Figure, A), consistent with progressive massive fibrosis, confirmed by computed tomography (Figure, B).

After infectious causes, including tuberculosis and histoplasmosis, were excluded, the patient was diagnosed with chronic silicosis and referred for transplant evaluation.

A: Chest x-ray image of patient. Arrows indicate bilateral upper lung opacification. B: Computed tomography chest image. Mass-like fibrosis (arrows) and displaced trachea (star) are indicated.

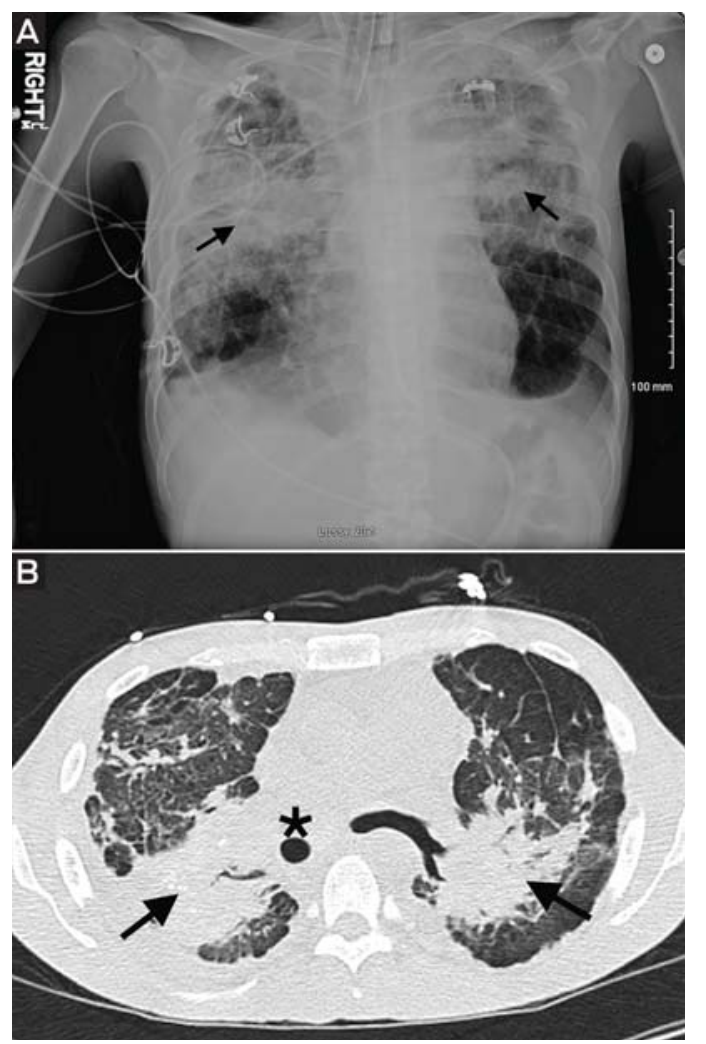

